Also publ. as paper in: International Conference on Computer Graphics and Interactive Techniques (SIGGRAPH), New Orleans, Louisiana, United States, August 1996, S. 148

\title{
Interactive Modelling of Branching Structures
}

\author{
Bernd Lintermann ${ }^{1}$ and Oliver Deussen ${ }^{2}$ \\ ${ }^{1}$ Institute for Dialog and Operating Systems, University of Karlsruhe, 76128 Karlsruhe, Germany \\ ${ }^{2}$ Institute for Simulation and Graphics, University of Magdeburg, 39016 Magdeburg, Germany
}

\begin{abstract}
We present a rule based modelling system that allows the graphical interactive definition of botanical structures such as plants, including partial and global constraints and free form deformations. The main idea is to represent the rule system by a structure tree with components of high functionality. In comparison to other rule based approaches, complex branching structures can be developed faster and more flexible.
\end{abstract}

The design of natural objects such as trees, bushes or flowers is a challenging task in two respects. One is the enormous structural complexity, the other is the large amount of geometrical properties that must be managed.

Different approaches have been introduced to create plants. Among formal techniques the most popular ones are textually edited grammars, known as L-systems [2]. Other specialized methods reduce the structural problem and concentrate on the generation of special objects such as trees [3].

In contrast to the rule based generation and subsequent graphical interpretation of letters in classical L-systems, our rule system is defined interactively by a graphical metaphor.

The software offers a set of predefined component types which play the role of letters in L-systems. Rules are created by combining components to a so called structure tree.
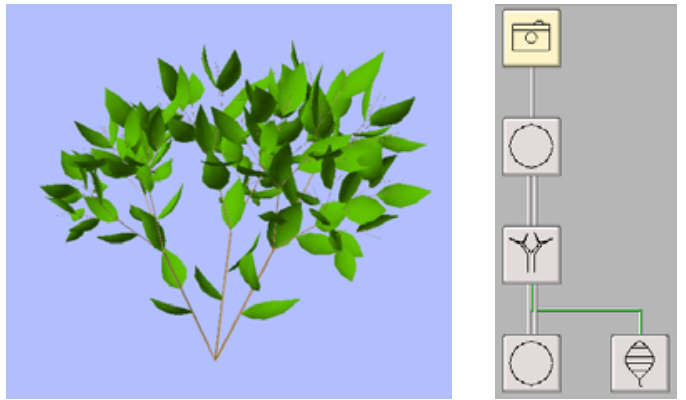

Figure 1: Bush with corresponding structure tree.

In the structure tree of figure 1, the camera component is the general root (the axiom). The component with the tree-like symbol produces a branch and defines the positions of subsequent leaves.

The component related to the circular symbol multiplies its successors and creates in this example three branches. The double line indicates a recursion. The leaf component defines the geometry of the natural leaves.

By double clicking on a component the corresponding parameters are displayed and can be changed. Each component defines at minimum a transformation towards its predecessor, a geometric primitive and a maximum recursion depth.
Specialized components place objects on curves and splines, provide lists of cross-sections that are triangulated in a post process or apply free form deformations or constraints to subsequent parts of the generated geometric data.

The effect of changing parameters is displayed immediately. Even complex structures $(\approx 100 \mathrm{k}$ triangles) are created and displayed on a SGI Indigo 2 Extreme in less than 5 seconds. Higher display rates can be achieved by reducing the displayed complexity during modelling or by hiding of components, which is supported by the system.

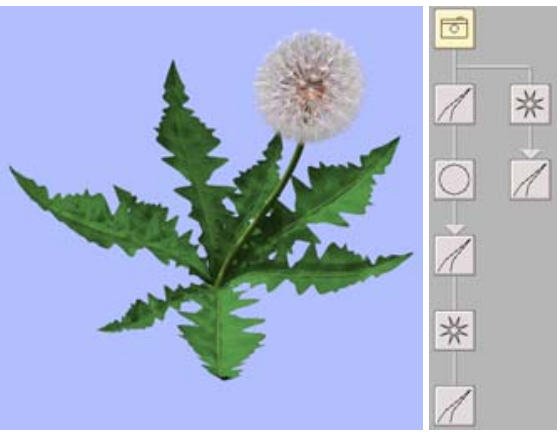

(a)

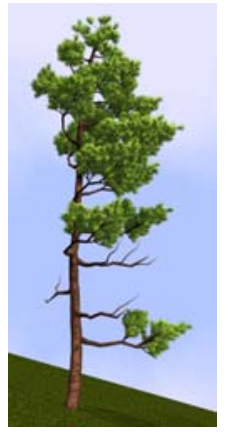

(b)
Figure 2: a) A dandelion (150k triangles) with structure tree; b) a pine under influence of wind.

The dandelion of figure 2(a) was created in two parts: the leaves are defined by splines and iterated around the stem (right part of the structure tree). The umbrellas are modelled by arranging hair on a rosette (lower three components of the left part), the head is done by placing umbrellas on a sphere according to the golden section ([1]).

The generation of the geometric data according to the structure tree is done as follows: the camera object defines the view and creates the iterator object for the leaves. By this object six leaf objects are created by rotating them around the origin. Also created by the camera is the stem. This object creates the iterator object which for its part creates the umbrellas and so on.

By introducing global fields, environmental factors can be modelled. This includes gravitropism, phototropism or the influence of wind. The pine of figure 2(b) was built using a horizontal tropism towards the right.

The software is availiable as a shareware, more information can be obtained by looking at URL: http://www.greenworks.de .

\section{REFERENCES}

[1] R. V. Jean. Mathematical modelling in phyllotaxis: The state of the art. Mathematical Biosciences, 64:1-27, 1983.

[2] P. Prusinkiewicz, A. Lindenmayer, and J. Hanan. Developmental models of herbaceous plants for computer imagery purposes. In J. Dill, editor, Computer Graphics (SIGGRAPH '88 Proceedings), Volume 22, Pages 141-150, August 1988.

[3] J. Weber and J. Penn. Creation and rendering of realistic trees. In Computer Graphics (SIGGRAPH '95 Proceedings), Pages 119-128, August 1995. 